\title{
Staatlich-nationale Erinnerungskultur in Griechisch-Thrakien
}

\author{
CAY LIENAU (Münster)
}

\section{Einleitung}

Erinnerungskultur wird von mir als formaler Oberbegriff verstanden für alle denkbaren Formen der bewussten Erinnerung bzw. des bewussten Erinnerns und Nichterinnerns an historische Ereignisse, Persönlichkeiten und Prozesse. ${ }^{1}$ Auf solche Formen des Erinnerns und Nichterinnerns - auch das Ausblenden von Personen und historischen Ereignissen gehört zur Erinnerungskultur - soll am Beispiel von Griechisch-Thrakien (= Westthrakien) eingegangen werden. In diesem ethnisch und religiös gemischten Gebiet werden spezifische Züge staatlich-nationaler Erinnerungskultur besonders deutlich.

Die Ausführungen beziehen sich v.a. auf die öffentliche (staatlich-nationale und kommunale) Erinnerungskultur, während auf die private Erinnerungskultur und die Traditionspflege der verschiedenen Volks- und Religionsgruppen nur am Rande eingegangen wird.

Behandelt werden soll das Thema an Beispielen von

- Orts- und Straßennamen

- Denkmälern

- Baudenkmälern und Museen

- der Darstellung des Raumes und seiner Geschichte in offiziellen Publikationen.

\section{Zur Geographie und Geschichte Griechisch-Thrakiens}

Im äußersten Nordosten Griechenlands gelegen, ist Griechisch-Thrakien Teil der historischen Region Thrakien, zu der große Teile des östlichen und südlichen Bulgarien und die europäische Türkei (= Ostthrakien) gehören. ${ }^{2}$

Im Westen bildet der Nestos (bulg. Mesta) die alte Grenze zu Makedonien, im Osten der Evros (bulg. Maritza) zur Türkei, im Norden der Haupt-

1 Vgl. dazu Cornelißen 2003, S. 555.

2 zur Geographie vgl. Schultze 1937. 
kamm der Rhodopen zu Bulgarien. Die in ihrem zentralen Teil über $2000 \mathrm{~m}$ hohen, nach Osten auf unter 1300 m Höhe abfallenden Rhodopen bilden das Rückgrat der Region. Eiserner Vorhang und auf griechischer Seite die Einrichtung einer Sperrzone, die nur mit Sondergenehmigung des Militärs betreten werden konnte und erst 1996 aufgehoben wurde, machten die Rhodopen $\mathrm{zu}$ einem undurchlässigen, schwer zugänglichen Gebiet und isolierten deren Bevölkerung.

Administrativ gliedert sich Westthrakien in die Verwaltungsbezirke (Nomoi) Xanthi, Rodopi und Evros. Die Mehrheit der Muslime lebt heute in den Bezirken Xanthi (Hauptort Xanthi) und Rodopi (Hauptort Komotini), während Evros (Hauptort Alexandroupolis) nur einen geringen Prozentsatz von muslimischer Bevölkerung besitzt (Tab. 1).

In dem von Thrakern bewohnten Gebiet gab es in der Antike mehrere griechische Siedlungskolonien, wie das 656 v. Chr. gegründete Abdera, Heimat von Protagoras und Demokrit, das im 7. Jh. v. Chr. von Chios aus gegründete Maroneia und Samothraki (das „thrakische Samos“). Wir können von regen Handels- und Kulturkontakten mit den thrakischen Stämmen ausgehen, die zwar als Barbaren, aber doch kulturell verwandt und (fast) auf gleicher Höhe stehend empfunden wurden. ${ }^{3}$

Teil des Oströmisch-Byzantinischen Reiches, wurde Thrakien bereits in der 2. Hälfte des 14. Jahrhunderts von den Osmanen erobert (also lange vor dem Fall von Byzanz 1453) und z.T. mit türkischer Bevölkerung aus Kleinasien besiedelt. Mit der Eroberung und Besiedlung durch die Türken beginnt zugleich eine Islamisierung dort lebender Bevölkerung. Am 11.8.1913 wurde Westthrakien vom Osmanischen Reich an Bulgarien abgetreten, im Zuge des Ersten Weltkrieges von den Alliierten bis Oktober 1919 besetzt und am 27.11.1919 Bulgarien entzogen. Mit dem Vertrag von Sèvres kam es am 10.8.1920 zusammen mit Ostthrakien an Griechenland.

Die „Kleinasiatische Katastrophe“, mit der für Griechenland 1922 der Krieg gegen die Türken in Kleinasien endete, führte mit den Verträgen von Lausanne 1923, die die Kriegsfolgen regelten, zu einer „ethnischen Bereinigung“ größten Ausmaßes: Alle Christen mussten die Türkei verlassen, sämtliche Muslime Griechenland (mit den unten genannten Ausnahmen). Waren die Umsiedlungen - in Grenzgebieten wurden z.T. ganze Ortschaften geschlossen umgesiedelt - zunächst noch freiwillig, so waren sie später obligatorisch. Annähernd 1,4 Mio. Christen verließen fluchtartig und unter schwierigsten Umständen die Türkei, ca. 0,4 Mio. Muslime Griechenland. ${ }^{4}$ Ihre Ansiedlung stellte insbesondere Griechenland vor größte Probleme.

Ausgenommen von dem Austausch blieben die Muslime Westthrakiens als Kompensation dafür, dass die Griechen in Istanbul/Konstantinopel, auf einigen

3 Vgl. Der Kleine Pauly 5, 1975, s.v. Thrake.

4 Vgl. Ladas 1932, S. 13, Sundhaussen 2001, S. 18, Kunz 1925. 
Inseln im Marmarameer und vor dem Ausgang der Dardanellen in das Ägäische Meer verbleiben konnten.

Wichtig für die Einschätzung der staatlich-nationalen Erinnerungskultur in Westthrakien ist die Tatsache, dass die Verträge von Lausanne 1923 die auszutauschende Bevölkerung nicht nach Sprache, sondern nach Religion unterschieden. So befanden sich unter den Menschen, die die Türkei verlassen mussten, neben christlichen Griechen christliche Albaner, Gagausen (türkisch-sprachige Christen) und Armenier, unter denen, die Griechenland verlassen mussten, neben Türken auch albanisch- und griechisch-sprachige Muslime.

Aufgrund der Tatsache, dass das griechische Westthrakien von der ethnisch-religiösen „Bereinigung“ ausgenommen blieb, finden wir dort noch jene ethnisch gemischten Strukturen, wie sie für das Osmanische Reich charakteristisch waren, allerdings nicht von der gleichen Art, wie sie vor den Balkankriegen dort herrschte. Bereits im Vertrag von Neuilly 1919, der die Beziehungen zu Bulgarien regelte, hatte man einen Bevölkerungsaustausch mit Bulgarien vereinbart, in dessen Folge nicht nur die meisten Bulgaren, die in der Region lebten, das Land verließen, sondern auch viele Türken v.a. im Grenzgebiet zur Türkei. ${ }^{5}$ Umgekehrt wurden Zehntausende von Flüchtlingen (Umsiedlern) aus dem Gebiet der Türkei, v.a. aus Ostthrakien und vom Schwarzen Meer (Pontosgebiet) in planmäßig angelegten Dörfern in Griechisch-Thrakien angesiedelt.

Heute sind dort ca. ein Drittel der Bevölkerung Muslime. Die Tatsache, dass Westthrakien bereits 1913 dem Osmanischen Reich verloren ging, bewirkte, dass es die von Atatürk in der Türkei durchgesetzten laizistischen Reformen zunächst nicht mitmachte, dann nur verzögert und unvollständig nachholte. Es blieb somit konservativer als die Türkei, was durch die nun eingetretene Minderheitensituation - aus dem bis dahin herrschenden Volk war eine Minderheit geworden, die sich unterordnen musste - befördert wurde.

Die Muslime sind ethnisch keineswegs einheitlich, sondern setzen sich zusammen aus

- Türken oder Turkophonen, den Nachkommen von Einwanderern aus Anatolien und anderen Teilen des Osmanischen Reiches,

- Pomaken (slavophone muslimische Volksgruppe),

- Zigeunern (turkogyftoi) islamischen Glaubens.

Auch die christliche Bevölkerung setzt sich aus verschiedenen Volksgruppen zusammen:

- alteingesessenen Griechen und deren Nachkommen, die v.a. in einigen Siedlungen an der Küste, auf den Inseln und in den Städten leben,

5 Vgl. de Jong, 1980a, S. XI. 
- Flüchtlingen, die nach der „Kleinasiatischen Katastrophe“ aus Ostthrakien, dem Pontosgebiet und anderen Teilen Kleinasiens dort angesiedelt wurden,

- aus dem übrigen Griechenland zugewanderten bzw. dorthin versetzten Griechen

- christlichen Roma und

- Russlandgriechen, die ab 1990 aus dem Kaukasus und dem Schwarzmeergebiet nach dem Zusammenbruch der Sowjetunion - den Russlanddeutschen vergleichbar - nach Griechenland zurückkehrten bzw. dorthin auswanderten und die vom griechischen Staat vornehmlich in Thrakien angesiedelt wurden, um dort das christliche Element gegen das muslimische zu stärken.

Die räumliche Verteilung der muslimischen Volksgruppen zeigt deutliche räumliche Schwerpunkte (Tab. 1).

Tab. 1: Räumliche Verteilung der Muslime in Westthrakien

\begin{tabular}{|c|c|c|c|c|c|c|c|c|c|}
\hline & 1 & 2 & 3 & 4 & 5 & 6 & 7 & 8 & 9 \\
\hline Xanthi & 103.300 & 42.000 & 40,8 & 10.000 & 9,7 & 23.000 & 22,3 & 9.000 & 8,7 \\
\hline Rodopi & 90.500 & 62.000 & 68,5 & 42.000 & 46,4 & 11.000 & 12,2 & 9.000 & 9,9 \\
\hline Evros & 143.800 & 10.000 & 7,0 & 2.000 & 1,4 & 2.000 & 1,4 & 6.000 & 4,2 \\
\hline W-Thr. & 337.600 & 114.000 & 33,8 & 54.000 & 16,0 & 36.000 & 10,7 & 24.000 & 7,1 \\
\hline
\end{tabular}

1. Einwohner 1991, 2. Muslime, 3. \% Muslime an Einwohnern, 4. Türken, 5. \% Türken an Einwohnern, 6. Pomaken, 7. \% Pomaken an Einwohnern, 8. Zigeuner, 9. \% Zigeuner an Einwohnern. Quelle: I Kathimerini vom 14.3.1993 und Nat. Stat. Serv. Greece 1995.

Die Lausanner Verträge hatten für den griechischen Staat zur Konsequenz, dass er die Minderheitengesetze unterschiedslos auf die Muslime in Thrakien anwenden konnte. Sie waren griechische Staatsbürger muslimischer Religion, denen man nach den Minderheitengesetzen zwar eigene Schulen mit Türkisch als Unterrichtssprache, das niedere Gerichtswesen und einige andere Rechte zugestand, nicht aber die Pflege nationaler Eigenständigkeit und damit einer eigenständigen Erinnerungskultur. Griechenland musste fürchten, dass eine ausgeprägte eigene Erinnerungskultur von bulgarisch-sprachigen Pomaken und Türken den Irredentismus förderte. Dies zu verhindern lag wahrscheinlich dem Geist der Zeit und ihrer führenden Politiker (Venizelos, Atatürk) entsprechend - schon in der Absicht der Verträge von Lausanne.

Der griechische Staat hielt die Minderheitengesetze zwar ein, handhabte diese aber seit den 50er Jahren des 20. Jahrhunderts bis in die 90er Jahre hinein restriktiv. Das Verhältnis zur Minderheit wurde bestimmt von der Lage der griechischen Minderheit in der Türkei und der türkischen Politik, die ihre Minderheit in Griechenland als Speerspitze im Fleische des Feindes betrachtete.

Auch wenn seit den 1990er Jahren deutliche Verbesserungen in der Behandlung der Minderheiten zu verzeichnen sind, werden von dieser immer noch mancherlei Benachteiligungen beklagt, so z.B. dass 
- die Angehörigen der türkischen Minderheit nicht das Recht genießen, sich auf Grund ihrer kulturellen Zugehörigkeit zu versammeln;

- Aktivitäten des türkischen Jugendverbandes von Komotini, des türkischen Bundes von Xanthi und des Verbandes türkischer Lehrer in Westthrakien nicht zugelassen werden, auch wenn es sich nur um Volkstänze, Nähkurse und dergl. handelt. ${ }^{6}$

Andererseits ist es jetzt erlaubt, von „Türken“ zu sprechen (früher durfte man nur von „Turkophonen“ reden und schreiben). Von Griechenland wurde neuerdings auch - wohl viel zu spät, um die Türkisierung noch aufzuhalten ein Wörterbuch der pomakischen Sprache herausgegeben, das die Grundlage für eine eigene pomakische Schriftsprache und ein pomakisches Bewusstsein (die es bisher nicht gab) und damit auch eine schriftliche Erinnerungskultur bilden soll. Heftige Kritik allerdings richtet sich dabei gegen die Verwendung des griechischen Alphabetes, das wenig geeignet erscheint, die Laute der pomakischen (bulgarischen) Wörter abzubilden.

Für den griechischen Staat bleiben die ethnisch unterschiedlichen muslimischen Volksgruppen auf der Grundlage der Verträge von Lausanne - aus politischen Gründen - eine immer noch als Einheit zu behandelnde Gruppe von Muslimen.

Vor diesem Hintergrund müssen die folgenden Ausführungen verstanden werden.

\section{Ortsnamen}

Die Benennung von Ortschaften ist in vielen Staaten Teil nationaler Erinnerungskultur. Man denke nur an die vielen Umbenennungen in den ehemaligen sozialistischen Staaten: Königsberg in Kaliningrad, Chemnitz in Karl-MarxStadt usf.

Anders als in diesen Fällen erfolgte in Griechenland mit der staatlichen Selbständigkeit jedoch eine planmäßige Umbenennung aller Ortschaften (wie etwa in Südtirol nach dem Ersten Weltkrieg). In Westthrakien geschah die planmäßige Umbenennung nach der Einbeziehung dieses Landesteiles in den griechischen Staat. ${ }^{7}$

Erste Bemühungen zur Umbenennung der in Griechenland derzeit gebrauchten Ortsnamen von Seiten des Staates erfolgten bereits 1843 per königlicher Verordnung und der Einsetzung einer Kommission zur Auffindung antiker geographischer Namen und der Namen berühmter Männer der antiken und neueren Geschichte, die zur Wiederherstellung bzw. Ersetzung bis dahin gebrauchter

6 http:www.diplomatischerbeobachter.com/news vom 21.6.2010: Die kulturellen Rechte der türkischen Minderheit in Westthrakien.

7 Die nachstehenden Ausführungen zur Geschichte der Ortsnamengebung in Griechenland folgen weitgehend Chatzidimitriou 1992. 
Namen geeignet erschienen. Die Kommission beschränkte sich dabei nicht allein auf die Ersetzung fremder Namen, sondern sollte auch die aktuellen griechischen Ortsnamen ändern, die nicht den Regeln der Katharevousa (der sog. ,reinen“ Sprache im Unterschied zur Dimotiki, der Volkssprache) entsprachen. Den Bemühungen fehlte allerdings eine wissenschaftlich abgesicherte Basis. Man vergab altgriechische Namen, ohne dass erwiesen war, dass die antiken Stätten, nach denen eine Ortschaft (um)benannt wurde, an der Stelle der umbenannten Ortschaft oder doch wenigstens in deren Region lagen. Gegen den Willen der Bevölkerung, deren Zustimmung man nicht einholte, wurden auch Orte umbenannt, an die sich Erinnerungen an die Freiheitskriege knüpften (z.B. Arachowa).

Am Ende der ersten Dekade des 20. Jahrhunderts setzte die Regierung im Rahmen einer Systematisierung der Ortsnamen (dies war für eine Volkszählung wichtig) eine neue Kommission zur Revision von Umbenennungen ein. Der Kommission gehörten Wissenschaftler und Verwaltungsbeamte an. Sie arbeitete mit örtlichen Kommissionen zusammen, die man zu diesem Zweck gebildet hatte. Die Kommission hatte zwei Ziele: die Ersetzung der schlecht klingenden und nicht mit besonderen Ereignissen der griechischen Geschichte verbundenen Namen und die Ersetzung früher vergebener ungeeigneter $\mathrm{Na}-$ men.

Mit dem Hinzukommen von Epirus und Makedonien zum griechischen Staatsgebiet im Jahr 1913 unternahm der Rat der Archäologischen Gesellschaft die Initiative für die Neueinsetzung einer Kommission, deren Aufgabe es war, altgriechische Ortsnamen zu sammeln und zu lokalisieren, die für eine Ersetzung aktueller Namen in Frage kamen.

Die mit dem Gesetz zur Einrichtung von Gemeinden im Jahr 1912 ganz in die Verantwortung der Gemeinden übertragene Aufgabe der Namensänderungen bewährte sich nicht, da zu viele Doppelbenennungen und andere Fehler vorkamen. Sie ging deshalb bald wieder in die Hände einer übergeordneten Kommission über.

Auch in Thrakien war eine zentrale Kommission in Zusammenarbeit mit örtlichen Kommissionen für die Umbenennung verantwortlich. Ein Rundschreiben ihres Sekretärs Stilpon Kyriakides, eines renommierten Professors für Volkskunde (laographia) an der Universität Thessaloniki, vom 10.10.1919 an die Gemeinden des Landes unterstrich, dass es deren Aufgabe war, die fremden Ortsnamen nach den zuvor in Griechenland angewendeten Regeln zu ersetzen. Er mahnte dabei an, sorgfältig bei den Ortsumbenennungen vorzugehen und Fehler zu vermeiden, die früher gemacht worden waren. Als Beispiel nannte er die Umbenennung von Gümülcine in Komotini statt in Gjoumourtzina, da es von der byzantinischen Familie der Koumoutzini herzuleiten sei. $^{8}$

8 Chatzidimitriou 1992, S. $71 \mathrm{ff}$. 
Priorität wird bei einer Umbenennung 9 altgriechischen Ortsnamen gegeben, die entweder direkt auf einen Ort an gleicher Stelle in der Antike bezogen werden können oder auf Orte in der Nähe, wie Xanthi oder Avdira (in der Antike Abdera).

Die Darstellung der Geschichte der Umbenennungen zeigt, wie wichtig dem griechischen Staat einerseits die Ausmerzung fremder Ortsnamen, die an die osmanische Vergangenheit erinnerten, andererseits die Anknüpfung an die Antike war.

Die Umbenennung (= Gräzisierung) der Ortsnamen folgt - wenn kein altgriechischer Bezug hergestellt werden kann - verschiedenen Mustern:

1. Die Siedlungsnamen werden aus dem Türkischen in das Griechische (oft recht frei und phantasievoll) übersetzt, so wird Akpinar (= weiße Quelle)

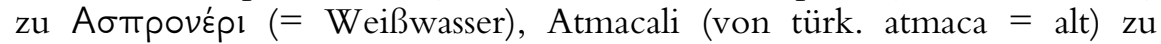
Г'́pokas (= Altdorf), Karacahalil (= ein Ort den Rehe mögen) zu

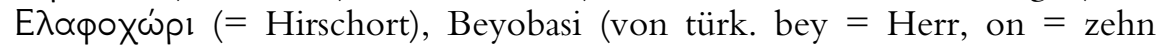
und bas $=$ groß) zu $\Delta \dot{\varepsilon}$ k $\propto \rho \times \circ$ (Dekarchon $=$ Zehn Herrscher).

2. Die Umbenennungen enthalten einen Bestandteil des alten Namens. So wird z.B. Çay Machale (çay $=$ kleiner Fluss) zu Pвú $\mu \alpha$ (= Fluss).

3. Es erfolgt eine indirekte Übersetzung des alten Namens: Kuru Köy (türk. kuru $=$ griech. xeros $=$ trocken) wird zu 'Aкарто (= das Unfruchtbare),



4. Umbenennungen lassen den alten Namen anklingen, eine Form assoziati-

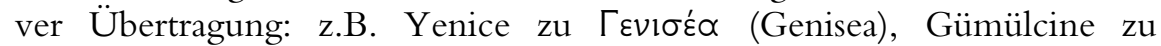

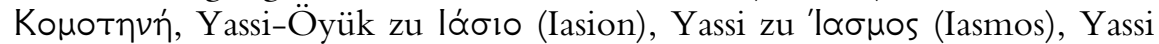
Ören zu Spaíov (Oraion). In Südtirol wurde auf diese Weise Bozen zu Bolzano, Auer zu Ora.

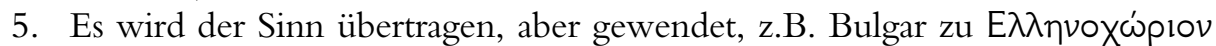
(= Griechenort). In Ostthrakien machte man aus Rumbeyli ein Islambeyli. ${ }^{10}$

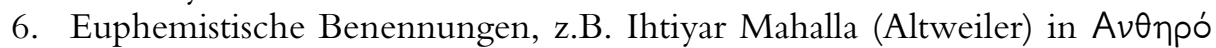
(= Blumenort).

7. Willkürliche Umbenennungen (soweit erkennbar), z. B. Ayvacik in $\Lambda i ß \propto s$ (= Schirokko).

8. Wiedergabe des alten Namens in griechischer Form, wobei der türkischen Benennung vermutlich bereits ein griechischer Name zugrunde lag. So wird


Chalep zu X $\propto \lambda \varepsilon \dot{\varepsilon r} \mathrm{l}$ (Chalepi).

Der Prozess der amtlichen Umbenennung war 1928 so gut wie abgeschlossen. ${ }^{11}$ Spuren der thrakischen und slawischen Siedlungsschicht lassen sich nur

9 Dazu Kreiser 1975 und 1978, Chatzidimitriou 1992.

10 Kreiser 1978, S. VIII. 
noch rudimentär erkennen. Im Sinne nationaler Erinnerungskultur muss Westthrakien heute als griechisch erscheinen.

Mit den Flüchtlingen nach der kleinasiatischen Katastrophe wurden viele Siedlungen neu gegründet. Sie erhielten, wenn die Siedler überwiegend aus demselben Ort kamen, den Namen des Herkunftsortes in griechischer Version mit dem Zusatz Nea oder Neon, wie z.B. Nea Kessani südöstlich von Xanthi am Westufer des Vistonissees (vor 1940 Plastiria, bis 1961 Nea Kissani), deren Bewohner großenteils aus dem ostthrakischen Keschan (griech. Kessani oder Kissani) und deren Umgebung kamen. Wir kennen die Übernahme des $\mathrm{Na}$ mens der Herkunftsorte oder Regionen z.B. aus Athen, wo die von Flüchtlingen besiedelten Stadtteile Nea Ionia oder Nea Smyrni hießen. Bisweilen wählte man aber auch so nichtssagende Bezeichnungen wie $\mathrm{N} \varepsilon 0 \times \omega \dot{\rho}$ l (= Neudorf) ${ }^{12}$, oder $K \propto \lambda \lambda_{\imath} \theta \theta^{\prime} \propto$ (= Schöner Blick). ${ }^{13}$

Zweisprachige Ortsnamenschilder werden vom griechischen Staat nicht zugelassen. Sie würden die Existenz nationaler Minderheiten einräumen.

Der Gebrauch der türkischen Ortsnamen ist in (griechischen) Druckerzeugnissen bislang verboten gewesen, so dass es auch keine offizielle griechische Schreibung gibt ${ }^{14}$, auch wenn die alten Ortsnamen bei der Bevölkerung durchaus noch bekannt und z.T. im Gebrauch sind.

In der Presse der westthrakischen Minderheit sind neben den türkischen Ortsnamen, die in phonetischer Transkription gebracht werden, immer die entsprechenden griechischen Namen anzugeben. ${ }^{15}$

\section{Straßennamen}

Wie Ortsnamen so sind auch Straßennamen Bestandteile der öffentlichen Erinnerungskultur. Die Namengebung erfolgt in der Regel durch den Stadtbzw. Gemeinderat. Mit der Namengebung werden Persönlichkeiten geehrt, die sich um die Stadt verdient gemacht haben oder von nationaler Bedeutung sind, es wird an Ortschaften (Städte, Regionen) erinnert, zu denen meist besondere Beziehungen bestehen oder an die erinnert werden soll, weil sie verloren gegangen sind, oder sie werden - in Griechenland häufig - christlichen Heiligen oder Gestalten der Antike gewidmet. Mit einer Veränderung der politischen und gesellschaftlichen Bedingungen erfolgte und erfolgt noch immer oft eine Umbenennung der Straßen. Da in osmanischer Zeit in Xanthi

11 Kreiser 1978, S. VIII. Zum Muster der Umbenennung von Ortschaften bei den Osmanen vgl. Wittek 1935, S. 11-64.

12 Nach Stamatelatos und Stamatelatou 3, S. 61 f. kommen sie über 60-mal in Griechenland vor.

13 Nach denselben Autoren 2, S. 21 f. über 40-mal in Griechenland.

14 de Jong 1980, S. XII.

15 Türkische Botschaft Berlin vom 2.3.2005. 
(das gilt für die anderen Städte im Osmanischen Reich vermutlich in gleicher Weise) nur wenige größere Straßen Namen hatten, handelt es sich bei den Straßennamen in dieser Stadt, die hier als Beispiel genommen wird, weitgehend um Neubenennungen.

Die wenigen Namen tragenden Hauptstraßen (caddesi) im osmanischen Xanthi waren v.a. nach Städten benannt, zu denen sie führten: Gümülcine caddesi, Istanbul caddesi, Kavala caddesi. Eine Straße hieß Hamam caddesi (= Badstraße). Nur die nach dem kaymakam Hüsseyin Effendi benannte Straße machte eine Ausnahme. Wenige Nebenstraßen bzw. Gassen (sokagi) hatten eigene Namen, wie Resit Beg sokagi oder Uzun sokagi (= Lange Straße). Ansonsten erfolgte die Lagebezeichnung nach den Wohnvierteln (mahalla): Sünne mahallesi, Cukur mahallesi, Yeni mahalla usf. Die Bezeichnung der Viertel wiederum folgt in der Regel dem Namen der zum Viertel gehörenden Moschee, der Bevölkerung, die dort wohnte (z.B. Yukari mahalla) oder bestimmten Eigenschaften bzw. Funktionen (z.B. Tabakhane mahallesi). Die Moscheen haben - anders als es bei den christlichen Kirchen der Fall ist -, keine Namenspatrone, sondern wurden nach Eigenschaften benannt (z.B. Yeni dschami) oder nach dem Viertel, in dem sie lagen. Die meisten der alten StraBennamen sind zumindest der älteren muslimischen Minderheitenbevölkerung, aber auch vielen älteren Griechen noch bekannt.

Wie die Ortsnamen so erfuhren die Straßennamen eine Umbenennung bzw. Neubenennung nach spezifischem Muster. ${ }^{16}$

Gruppiert man die aktuellen Straßennamen Xanthis nach den o.g. Gesichtspunkten, dann ergibt sich nach der Häufigkeit folgende Gruppierung:

1. Am häufigsten sind Straßennamen, die nach Persönlichkeiten benannt sind; davon fast zwei Drittel nach Persönlichkeiten des neuen Griechenland, knapp ein Drittel nach Personen aus der Antike, nur ca. 8\% nach solchen aus der byzantinischen Zeit. Beispiele sind Odos (= Straße) Athanasiou Diakou nach dem Helden der Freiheitskriege Athanasios Diakos (1788-1821), Odos Aischylou nach dem antiken Tragödiendichter oder Odos Agiou Georgiou nach dem heiligen Georg. Der Name der Straße steht dabei immer im Genetiv.

2. Mehr als ein Viertel der Straßennamen bezieht sich auf Örtlichkeiten, insbesondere Städte. Über die Hälfte von ihnen sind Städte in ehemals von Griechen bewohnten Gebieten in Kleinasien (z.B. Odos Trapezountos, Odos Smyrnis) und Ostthrakien (z.B. Odos Arkadiou), drei auch aus dem von einer griechischen Minderheit bewohnten Nordepirus in Albanien (z.B. Odos Argyrokastrou). Die Benennung einer Straße nach der deutschen Stadt Gifhorn geht auf die Partnerschaft Xanthis mit dieser Stadt zurück, wobei vermutlich Menschen aus Xanthi und Umkreis dort als Gastarbeiter tätig waren.

16 Vgl. dazu Avramidou-Kemanetzi / Aivaliotis 2003 und Dimos Komotinis 1994. 
3. Etwa 10\% der Straßennamen beziehen sich auf Ereignisse, von denen die meisten in die Zeit des neuen Griechenland fallen, z.B. Odos Tetartou Oktovriou 1919, d.h. Straße des 4. Oktober 1919 (Tag der Befreiung Xanthis von den Bulgaren), wenige auf die Antike (z.B. Odos Salaminas = nach der Insel Salamis, vor der die Griechen 480 v. Chr. die Perser in der berühmten Seeschlacht schlugen).

4. Ein kleiner Rest bezieht sich auf andere Dinge.

Kein einziger Straßenname erinnert jedoch an einen muslimischen Gelehrten, Geistlichen, eine muslimische Stadt oder überhaupt an die Existenz von Türken und Pomaken. Die Namen der an Moscheen vorbei führenden Straßen folgen dem beschriebenen Muster.

Die Erinnerung im Straßennamen an die Antike, die byzantinische Zeit und die Zeit der Freiheitskriege soll dabei ebenso Identität stiften wie die Erinnerung an Orte und Regionen, aus denen viele Flüchtlinge nach 1922 kamen. Wohl in Ermangelung von griechischen Persönlichkeiten, die aus Thrakien stammen, finden sich nur wenige thrakische Persönlichkeiten als Namengeber, sieht man von einigen Bürgermeistern Xanthis ab.

Die Art der Namengebung erinnert an die Motive auf griechischen Briefmarken, die ebenfalls ein Teil Identität stiftender Erinnerungskultur sind.

\section{Exkurs zu griechischen Briefmarken}

Briefmarken sind in fast allen Staaten der Erde staatliches Monopol und dienen bzw. dienten mit den auf ihnen abgebildeten Motiven und ihrem Design immer auch der Selbstdarstellung von Staaten, als Transmissionsriemen von Botschaften, die diese vermitteln wollen. Sie sind Ausweis national-staatlichen Identitätsbewusstseins. ${ }^{17}$

Auffälligstes Merkmal griechischer Postwertzeichen ist die überragende Rolle, die antike Motive und antikisierendes Design spielen. Bis 1912, also mehr als 50 Jahre nach Herausgabe der ersten griechischen Marken im Jahre 1861, wurden ausschließlich antike Motive verwendet in Form des sog. GroBen und Kleinen Hermeskopfes, ganzfiguriger Gestalten von Hermes und der Götterbotin Iris sowie - anlässlich der ersten Olympischen Spiele der Neuzeit 1896 in Athen und der sog. Zwischenolympiade 1906 - von (antiken) Sportmotiven und (antiken) Bauten. Erst mit dem sog. Ekstrateia-Satz, der 1912 in Erinnerung an den Sieg Konstantins an der Milvischen Brücke vor 1600 Jahren - sicher nicht ohne Absicht - am Ende des Ersten Balkankrieges zur Benutzung in den befreiten Gebieten herausgegeben wurde, erscheint ein christliches Motiv auf griechischen Briefmarken. Eine Marke des Satzes zeigt ein gleichschenkliges Kreuz im Strahlenkranz und darüber die Worte EN TOYTSI NIKA (in diesem Zeichen siege), wie es Konstantin vor der Schlacht an der

17 Vgl. Lienau 2002. 
Milvischen Brücke erschienen sein soll, darunter die Akropolis, die zum Symbol und Logo Griechenlands wird. Eine andere Marke des Satzes zeigt einen Adler mit Schlange im Schnabel unter den aus Wolken hervorbrechenden Strahlen der Sonne.

Mit christlichen Themen und christlicher Symbolik macht der EkstrateiaSatz von 1912 den Anfang eines weiteren wichtigen Motivbereiches: Jetzt wird auch die byzantinisch-christliche Vergangenheit als Glied in die bis zur Antike zurück gefuihrte Tradition einbezogen und - da v.a. die Kirche in der Zeit der Osmanenherrschaft Träger der griechisch-christlichen Kultur war eine lückenlose Kontinuität zur Gegenwart hergestellt. ${ }^{18}$ Das späte Erscheinen christlich-byzantinischer Motive auf Briefmarken spiegelt das späte Zurückgreifen auf die christlich-byzantinische Vergangenheit als Wurzel staatlichen Selbstverständnisses.

Auffallig ist andererseits das totale Ausblenden der als Trauma empfundenen über 500 Jahre währenden osmanischen Vergangenheit, die dazu führt, dass z.B. bei den Marken von Xanthi und Komotini in der Serie der Hauptorte der 53 Präfekturen (Nomoi) anstelle der für das Stadtbild charakteristischen muslimischen Bauwerke untypische Bauwerke nicht-muslimischen Charakters dargestellt werden.

Während andere Staaten auf ihren ersten Briefmarken schlichte Zahlenwerte oder das Portrait des Herrschers abbilden (so auf den ersten englischen, preußischen oder frühen bayerischen Postwertzeichen), weist Griechenland mit den antiken Motiven nicht nur auf seine Wurzeln, sondern verbindet sich mit Europa, dessen philhellenische Strömungen nicht wenig zur staatlichen Selbständigkeit beigetragen hatten. Die Antike - das dokumentieren die Briefmarken - ist an erster Stelle, die byzantinisch-christliche Vergangenheit an zweiter Stelle für Griechenland identitätsstiftend.

In dem Rückbezug auf Antike und byzantinische Vergangenheit spiegelt sich das Bedürfnis des griechischen Staates, die neue und fragile Identität durch Rückbezug auf die vorosmanische Zeit historisch zu begründen. Er unterscheidet sich darin nicht von den anderen Balkanstaaten, die alle den Versuch unternehmen, die eigene Kultur und Identität durch Bezug auf die vorosmanische Vergangenheit zu bestimmen und zu legitimieren. ${ }^{19}$ Dieser Rückbezug stellt einen Grundpfeiler des griechischen Identitätsbewusstseins und griechischer Identitätssuche dar. ${ }^{20}$

Mit dem Bezug auf die Antike und die christlich-byzantinische Vergangenheit postuliert der Staat eine lange historische Kontinuität, eine Kontinuität, die zwar äußerlich durch die Jahrhunderte währende Türkenherrschaft unterbrochen wurde, innerlich aber durch die sprachliche und kirchliche Kontinuität erhalten blieb.

18 Vgl. Hering 1989, S. 330.

19 Vgl. ebd., S. $340 \mathrm{ff}$.

20 dazu Tzermias 1994. 
In der kurzen Zeit zwischen 1913 und 1919, in der Westthrakien eigene Briefmarken hatte, wurden dort griechische und türkische Marken mit Überdruck verwendet, ein deutliches Indiz für unklare Identität. ${ }^{21}$

\section{Denkmäler}

Griechenland ist ein denkmalfreudiges Land: auf nahezu jeder Platia (zentraler Platz), in jedem Park findet man Denkmäler, die an Freiheitskämpfer, Dichter, Denker und Wohltäter von nationaler oder auch nur lokaler Bedeutung erinnern. Denkmäler sollen integrierend wirken, die Einheit der Nation erzeugen. ${ }^{22}$ Sie sind Repräsentationsmodi, die ein Staat als Bedeutungsträger nutzt, um die Orientierung, die Kultur, das Recht und die Herrschaft der Nation mit der Geschichte des Volkes zu verbinden und somit ein spezielles Bewusstsein der gemeinsamen Geschichte zu erschaffen. ${ }^{23}$ Wie bei den Straßennamen bestimmt eine Kommission über die Errichtung von Denkmälern. Die Kommission setzt sich aus Vertretern des Staates und/oder der jeweiligen Stadt bzw. Kommune zusammen.

Eine Durchmusterung der Denkmäler in Thrakien zeigt, dass in dieser ethnisch-religiös gemischten Region eine gewisse Rücksicht auf die Minderheitensituation zu spüren ist.

Es fällt auf, dass in vielen muslimischen Dörfern (besonders den pomakischen) Denkmäler fehlen, dass in den Bezirken Xanthi und Rodopi, in denen der Anteil der muslimischen Minderheit weitaus größer ist als im östlichsten thrakischen Nomos Evros, viele Denkmäler unspezifiziert den Kriegstoten oder - meist sonst wenig bekannten - Kriegshelden der jüngeren gemeinsamen Vergangenheit nach 1920 gewidmet sind, während Denkmäler für die Helden der Freiheitskämpfe 1821 ff., die im übrigen Griechenland allgegenwärtig sind, fehlen.

Die unspezifizierte Widmung vieler Denkmäler an Kriegstote lässt offen, auf welcher Seite die Toten gestanden haben, da ja alle Seiten Kriegstote zu beklagen haben. Die offen gelassene Deutung eines Denkmals geht im kuriosesten Falle so weit, dass das Denkmal nur aus einem Sockel mit großer Marmorplatte ohne Inschrift besteht, wie das in dem von Muslimen und Christen (überwiegend christlichen Zigeunern) bewohnten Dorf Aratos östlich von Komotini der Fall ist (Abb.1, S. 413).

Selten erscheinen muslimische Namen auf Gedenktafeln, so auf dem durch Sprayer verunzierten - Denkmal für Gefallene im Heroenpark (Platia Pezonton Iroon) von Xanthi. Ob es sich bei den Namen um die Namen von Türken, Pomaken oder Zigeunern handelt, lässt sich nicht erschließen. Die

21 Vgl. dazu Giannoudi 1975/76 und Galinos 1991.

22 Hettling in Koselleck / Jeismann 1994, S. 169.

23 Lipp 1999, S. 133. 
Gründe dafür, dass so wenige Muslime auf Gedenktafeln erscheinen, mögen darin liegen, dass das muslimische Element staatlicherseits bewusst ausgeklammert wird. Manche Muslime mögen im Bürgerkrieg auch auf der „falschen“ (kommunistischen) Seite gestanden haben und fanden deshalb keine namentliche Ehrung (die Mehrzahl der Muslime stand allerdings auf der konservativen Seite). Leider waren keine Zeugnisse der vermutlich zur Einweihung der Denkmäler gehaltenen Reden zu beschaffen, so dass die Deutung Spekulation bleiben muss.

Denkmäler, die bewusst in Inhalt und Gestaltung Bezug auf die griechische Antike nahmen, sind selten, fehlen aber nicht ganz.

Ein schönes Beispiel dafür ist das Denkmal für neun von Deutschen 1941 eingeschlossene und im Kampf gefallene griechische Soldaten am Ortseingang von Echinos, dem Hauptort der Pomaken in den Rhodopen (Abb. 2, S. 414). Nicht nur die Amphore weist auf die Antike; auch die Inschrift nimmt darauf Bezug, indem es den aussichtslosen Abwehrkampf der Soldaten mit dem der Spartaner vor 2500 Jahren bei den Thermopylen vergleicht:

KAI PRAGMATI PETHANAN ANTIGRAFONTES EPEITA APO 2.500 CHRONIA PERIPOU TIN ISTORIAN TON THERMOPYLON.

Wie wenig allerdings solche Denkmäler das Identitätsbewusstsein der dort lebenden Pomaken offenkundig beeinflussen, zeigt eindrucksvoll ein kleines Denkmal Alexanders d. Gr., das einem Dorf gestiftet worden war und das man mangels eines in der Öffentlichkeit akzeptierten oder geeignet erscheinenden Platzes an den Eingang der örtlichen (von griechischen Polizisten besetzten) Polizeistation gestellt hatte. Direkt davor auf der Plateia sitzende Muslime konnten unsere Frage, wer das sei, (im Frühjahr 2006) nicht beantworten.

Fazit: Augenfällig ist in der Denkmalkultur der westthrakischen Bezirke Xanthi und Rodopi eine gewisse Rücksichtnahme auf die muslimische Minderheit, auch wenn diese generell aus der Denkmalkultur ausgespart und die über fünfhundertjährige Epoche, in der die Region zum Osmanischen Reich gehörte, ausgeblendet wird.

\section{Baudenkmäler, Ruinenstätten, Denkmalpflege, Museen}

Baudenkmäler, zu denen weltliche und sakrale Bauten in den Städten und Dörfern gehören, aber auch Ruinenstätten und Museen sind Fenster in die Vergangenheit. Der Umgang mit ihnen wirft ein Licht auf die jeweilige Gesellschaft und deren Erinnerungskultur.

Thrakien ist nicht gerade reich an kunsthistorisch bedeutenden Baudenkmälern, aber reich an baulichen Zeugnissen aus der Vergangenheit, die die wechselvolle Geschichte des Raumes abbilden. Da sind Ruinenstätten aus der Antike, z.B. die Ruinen des antiken Samothrake, wo die berühmte Nike, die sich jetzt im Louvre in Paris befindet, gefunden wurde (als Kopie ist sie in dem 
kleinen Museum der Ausgrabungsstätte zu sehen), die Ruinen des antiken Abdera (Avdira) an der Küste gegenüber der Insel Thasos, wo der berühmte Philosoph Demokrit lebte, oder die Reste der wieder frei gelegten Via Egnatia. Bauwerke aus byzantinischer Zeit sind als Brücken in den Rhodopen, Festungsbauwerke und Kirchen erhalten. Bauwerke aus osmanischer Zeit erhielten sich als Bürgerhäuser in den Städten, als Moscheen und andere religiöse Bauwerke (Medresen, Tekkedes), aber auch als christliche Kirchen und weltliche Bauwerke wie z.B. die Tabakhäuser in Xanthi und der Leuchtturm in Alexandroupolis.

Im Sinne von Erinnerungskultur entscheidend ist der Umgang mit den baulichen Zeugnissen aus der Vergangenheit. Erst durch bewusste Pflege werden sie zu Baudenkmälern im eigentlichen Sinne; ihr Abriss oder ihre Preisgabe an den Verfall ist gewissermaßen negative Erinnerungskultur. Für Griechenland insgesamt gilt die bewusste Vernachlässigung aller Bauten, die erkennbar türkischen bzw. osmanisch-muslimischen Charakter tragen. Beispiel dafür ist das Jahrzehnte lang dem Verfall überlassene Imaret im benachbarten makedonischen Kavala (Abb. 3, S. 415, es ist seit wenigen Jahren zu einem Café und Hotel umgestaltet).

In Westthrakien blieben davon, weil gesetzlich geschützt, die Moscheen und mit ihnen verbundene Bauwerke ausgenommen, allerdings auch nicht vollkommen. Von der 1944 während der bulgarischen Besatzung abgerissenen großen Moschee im Zentrum Xanthis blieb nur der Uhrturm an der Platia Dimokratias als Wahrzeichen Xanthis stehen.

Noch nicht Teil der öffentlichen Erinnerungskultur und damit einer irgendwie gearteten Denkmalpflege wurden die zahlreichen und oft eindrucksvollen Ruinenstätten: Dorfwüstungen in den Rhodopen, die Zeugnis ablegen von der wechselvollen Geschichte dieses Raumes seit den Balkankriegen. Sie sind - sieht man von den jüngsten Wüstungsprozessen wie etwa in Alt-Xerias $\mathrm{ab}$ - Produkt der Grenzziehungen nach den Balkankriegen und dem Ersten Weltkrieg und den damit verbundenen Nationalisierungsprozessen und Bevölkerungsbewegungen. ${ }^{24}$

Ostmakedonien und Thrakien sind reich an wüst gefallenen Dörfern in den Rhodopen, die von ihren Bewohnern nach den Balkankriegen, nach dem in den Verträgen von Lausanne 1923 vereinbarten Bevölkerungsaustausch und später verlassen wurden. Mit ihrer Aufgabe verfiel auch die Flur.

Manche Ruinenstätten werden von ihren ehemaligen Bewohnern und deren Nachkommen zum Patronatsfest, zum 1. Mai oder 15. August aufgesucht. Die Treffen werden mit Gottesdienst, Tanz und Geselligkeit gefeiert. Die oft ausgedehnten Ruinenstätten verlassener Dörfer und die an Feldmauern, Wegen, Fruchtbäumen und anderem erkennbaren ehemaligen Feldfluren spielen in der Erinnerungskultur ihrer einstigen Bewohner und deren Nachkommen

24 Dazu Ladas 1931, S. 13 ff., auch de Jong 1980, S. XV. 
noch eine wichtige Rolle, in der staatlichen Erinnerungskultur dagegen keine. Das soll an den Beispielen der näher untersuchten Wüstungen von Alt Xerias und Ano Livera erläutert werden.

Mit der beispielhaften Kartierung der Wüstungen Ano Livera und Alt-Xerias und der Rekonstruktion vom Leben und Wirtschaften in diesen Siedlungen, bevor sie die Bewohnern verließen, sowie den Gründen ihrer Aufgabe durch Befragen von Zeitzeugen wurde in den Jahren 1996-2003 im Rahmen von Praktika unter Anleitung des Verf. versucht, den Grundstein für eine Einbeziehung der Wüstungen in die Erinnerungskultur Westthrakiens zu legen.

Die ausgedehnte Wüstung Ano Livera (Abb. 4, S. 415) liegt auf einer Terrassenfläche östlich oberhalb der Nestosschlucht in der Nähe des Ortes Stavroupolis. Die Hausruinen und erhaltenen Grundmauern der ehemaligen Höfe zeigen das Bild eines typischen muslimischen Dorfes mit Höfen, die durch Mauern gegen die Straßen abgeschirmt wurden. Wirtschaftliche Grundlage war der Tabakbau (darauf lassen brunnenartige Vertiefungen in den Ruinen einzelner Gehöfte schließen, die früher zur Feuchthaltung und Fermentierung der Tabakblätter dienten). Umgestürzte und zerbrochene Grabstelen markieren zwei muslimische Friedhöfe. Nach Verlassen des Dorfes siedelten sich griechische Flüchtlinge im östlichen Teil an und verwandelten die ehemalige kleine Moschee in eine christliche Kirche. Die Griechen verließen das Dorf jedoch recht bald (wohl spätestens in und nach dem Bürgerkrieg), so dass Dorf und Flur verwaisten. Ein Hirte bewohnt noch das Dorf. Ein ehemaliger Gastarbeiter aus Deutschland siedelte sich neu an (Beginn einer Wiederbelebung?) und baute sich in fränkischem Stil im oberen Teil der Dorfwüstung ein neues Haus. Die an Terrassenmauern, Feldeinfriedungen, Dreschtennen, verwilderten Fruchtbäumen und anderem erkennbare Flur wird heute von Schafen, einigen Rindern und einer Herde von verwilderten Pferden (die vermutlich beim Verlassen des Dorfes zurück blieben und sich in der Freiheit vermehrten) beweidet. Sie beschleunigen den Verfall der über Jahrhunderte geleisteten Kulturarbeit.

Das Schicksal von Alt Xerias (türk. Kouroudere $=$ trockener Fluss, das griechische Xerias ist eine freie Übersetzung), das westlich des Nestos oberhalb der neuen Via Egnatia (E 90) auf den Vorhügeln der Rhodopen liegt, ist ähnlich. Das Dorf, dessen Gemarkung bis an den Nestos reicht, wurde allerdings viel später als Ano Livera verlassen. Bis 1922 lebten dort türkische Muslime als recht wohlhabende Tabakbauern, wenn man die noch erhaltene Bausubstanz als Zeugnis dafür nimmt. Nach Verlassen durch die Türken zogen griechische Flüchtlinge in das Dorf, errichteten dort eine Schule und andere öffentliche Bauwerke neu und wandelten die Moschee in eine christliche Kirche um. Sie verließen den Ort Anfang der 70er Jahre des 20. Jahrhunderts, um sich an verkehrsmäßig günstigerer Stelle in der Ebene mit staatlicher Hilfe anzusiedeln. Die Bausubstanz ist aufgrund der noch nicht so lange wie in Ano Livera zurückliegenden Aufgabe wesentlich besser erhalten, aber auch sie verfallt zuse- 
hends. Das wird befördert durch griechische „Schatzsucher“, denn viele Türken hatten - wohl in der Hoffnung, einmal in ihr Dorf zurückkehren zu können - ihr Geld in Mauern und anderen Bauteilen versteckt, die nun von den Schatzsuchern zerstört werden.

Ano Livera wie Alt Xerias sind beeindruckende Ruinenstätten, deren Konservierung als Zeugnisse der jüngeren Geschichte und Attraktion für Tourismus durch den griechischen Staat indes in keiner Weise gefördert wird. Sie finden nicht einmal Erwähnung in offiziellen Publikationen, werden also in keiner Weise in die offizielle Erinnerungskultur einbezogen. Ob ihre - virtuelle - Rekonstruktion durch die wissenschaftlichen Arbeiten und deren Publikation dazu beitragen kann, muss vorerst dahin gestellt bleiben.

Die Bemühungen der Denkmalpflege konzentrieren sich eindeutig auf die Ausgrabung antiker Ruinenstätten und die mit Sanierung verbundene Renovierung von Altstadthäusern in den Städten. Die muslimischen religiösen Bauwerke werden von den Muslimen mit Geldern aus Stiftungen und ausländischer Hilfe unterhalten. Viele weltliche Bauwerke aus osmanischer Zeit gibt man, auch wenn sie kulturhistorisch und kunsthistorisch wertvoll sind, dem Verfall preis.

Abgesehen von den überregional bedeutenden Museen, die mit den antiken Ausgrabungsstätten von Samothraki und Avdira verbunden sind, haben die wenigen Museen v.a. heimatkundlichen Charakter. Die Osmanenzeit erscheint in ihnen, wenn denn die über 500 Jahre währende Zeit der Osmanenherrschaft überhaupt berücksichtigt wird, als Teil der Volkskultur. Sie wird damit ins Folkloristische gewendet, wenn ausgestellte Trachten, Tafelgeschirre und ähnliche Exponate als „traditionell“ bezeichnet werden. Beispiel dafür ist das schöne Volkskundliche Museum der Filoproodi Enosi Xanthis (= Union der Fortschrittsfreunde Xanthis) in Xanthi, das in zwei Häusern reicher griechischer Tabakhändler in der Altstadt Xanthis untergebracht ist. Es zeigt das Leben und Ambiente der Tabakhändler im Erdgeschoss, das bäuerliche Leben der Region im Keller und das Ambiente einer lokal bekannten Schriftstellerin im Obergeschoss.

Wenige Museen sind speziellen Themen gewidmet wie das Seidenmuseum in Soufli oder das vom jetzigen Metropoliten 1972 gegründete Bischöfliche Museum in Alexandroupolis mit einer Sammlung religiöser Gegenstände aus der Region. Es präsentiert eine Kultur, für die Alexandroupolis, das türkische Dedeagatsch, keinerlei kirchliche Tradition besitzt, da es erst nach 1922 Bischofssitz wurde. Es ist mehr als Zeichen der Präsenz des Christentums zu verstehen, ebenso wie der an markanter Stelle neben einer älteren kleinen Kirche errichtete mächtige Neubau einer orthodoxe Kirche in dem kleinen Ort Porto Lagos an der Europastraße 90.

Die „Philosophie“ der griechischen Baudenkmalpflege und Museumskultur erscheint als dieselbe wie in den zuvor erläuterten Punkten: die Baudenkmäler der Antike und der byzantinischen Zeit werden als Zeugnisse einer un- 
gebrochenen griechischen Siedlungskontinuität aufgefasst und dienen als Identifikationsmerkmale für das neue Griechenland. Wie sehr dies von der Bevölkerung verinnerlicht wurde, schildert de Jongh anlässlich eines Besuches der antiken Ruinen von Avdira:

Ein Fischer...sprach voller Stolz von seinen namhaften Landsleuten, v.a. von Demokrit. Er schilderte ihn so anschaulich, als wäre er erst vor ein paar Tagen und nicht schon um 370 v. Chr. gestorben. ${ }^{25}$

Die Restaurierung der Altstädte und ihrer bürgerlichen Bauten, die großenteils aus der Zeit der Osmanenherrschaft stammen, werden nicht als Zeugnisse jener Epoche, sondern als Zeugnisse traditionaler Volkskultur ausgewiesen. Was nicht hineinpasst, wird vernachlässigt oder ausgeblendet.

Das Verhalten der griechischen Behörden gegenüber dem türkischen kulturellen Element kann als

die Vernichtung und Ausradierung der von den türkischen Eroberern der einstmals byzantinischen Region hinterlassenen kulturellen Spuren und stattdessen die völlige Hellenisierung der Region ${ }^{26}$

beschrieben werden.

$\mathrm{Zu}$ verstehen ist dies nur aus dem „Osmanentrauma“, das Griechenland mit anderen Staaten Südosteuropas teilt.

\section{Thrakien in der Darstellung offizieller Publikationen}

Mit offizieller Literatur über Thrakien sind Bücher, Broschüren und Pläne gemeint, die von Ministerien, z.B. dem Tourismus-Ministerium in Athen, Kommunalverwaltungen oder anderen öffentlichen Institutionen über die Region, über Städte und Einzelgegenstände der Region herausgegeben wurden, um über das Land zu informieren und dafür zu werben.

Mustert man diese Literatur durch, dann ergibt sich - hier an wenigen Beispielen aufgezeigt - das gleiche Bild wie bei den zuvor behandelten Punkten:

- In allen vom Staat oder Kommunen herausgegebenen Büchern wird die über 500jährige Zeit der Osmanenherrschaft und die mit dieser Zeit verbundene Kultur übergangen, als dunkle Zeit ausgeblendet. Es findet keinerlei Auseinandersetzung mit der Zeit und deren kulturellen Leistungen statt. Zeugnisse der Kultur der osmanischen Zeit werden als „,traditionell“, „thrakisch“, ,frankolevantinisch“ oder „,muslimisch“, nie als ,türkisch“" oder ,pomakisch“ bezeichnet.

Das vom regionalen Generalsekretariat von Ostmakedonien und Thrakien (Generalsekretariat 1994) über die Kultur Westthrakiens veröffent-

25 de Jongh 1985, S. 124.

26 Quelle s. Anm. 6 
lichte über 400 Seiten starke Buch „Thrakien“ weist als Autoren Professoren der Archäologie und Byzantinischen Geschichte bis hin zu Professoren der Neugriechischen Geschichte, aber keinen einzigen Fachmann für die osmanische Zeit aus. In dem Buch nimmt die Osmanenzeit nicht einmal 20 Seiten ein, die fast ganz dem Griechentum Thrakiens in jener Zeit und dessen Rolle für die griechische Geschichte gewidmet sind.

- Viele Publikationen beginnen mit der Aussage, dass Thrakien seit sehr früher Zeit bzw. seit der Antike „hellenisch“ sei. Das ist die bekannte Herstellung einer Verbindung mit der Antike, die zugleich dazu dient, die Zugehörigkeit Westhrakiens zum griechischen Staatsgebiet zu legitimieren.

- In älteren Stadtplänen von Xanthi und Komotini sind zwar die christlichen Kirchen, nicht aber die Moscheen eingezeichnet.

Man kann in all diesen Fällen von gezielter oder manipulierter Erinnerungskultur sprechen.

\section{Fazit}

Die offizielle Erinnerungskultur in Thrakien knüpft ebenso an die Antike und byzantinische Vergangenheit an, wie es in Griechenland bereits vor der Einverleibung Thrakiens der Fall war. Antike und orthodoxes Christentum und deren Zeugnisse sind als gemeinsames Wissen und als gemeinsame Werte Identität stiftend im Bewusstsein der Bevölkerung geworden (wie sich dies u.a. in der Wahl der Vornamen bei den Griechen ausdrückt: die überwiegende Zahl der Vornamen sind die Namen antiker Personen oder christlicher Heiliger wie Sophoklis, Homiros, Georgios oder Dimitrios). ${ }^{27}$ Die Zeit der Osmanenherrschaft wird in der Erinnerungskultur zur dunklen Zeit, deren kulturelle Zeugnisse ausgeblendet oder zu einem folkloristischen Element abgewandelt werden. Die Erbitterung auf diese Zeit der Unterdrückung durch die Osmanen wird ebenso zu einem konstituierenden Element des neuen Griechenland wie die heroisch verklärte Antike und das als Brücke dorthin verstandene orthodoxe Christentum. Somit trägt die Erinnerungskultur auch kulturkämpferische Züge.

Dass Epochen der Vergangenheit vom Staat umbewertet oder deren Zeugnisse beseitigt werden, kennen wir auch aus der eigenen deutschen Vergangenheit oder sogar Gegenwart, wenn man an die Sprengung der Garnisonskirche in Potsdam oder des Berliner Schlosses in der DDR, den Abriss des Palastes der Republik der DDR im heutigen Zentrum Berlins oder auch die vielen Straßenumbenennungen denkt. Unterschiede ergeben sich in Art und

27 Vgl. dazu auch Assmann 1999, S. 16. 
Umfang solcher Aktionen, die ein spezifisches Licht auf die jeweils damit befassten Staaten und Gesellschaften werfen.

In Thrakien können wir einen etwas sensibleren Umgang mit Erinnerungskultur wahrnehmen, wenn man auf die Denkmäler im eigentlichen Sinne sieht und diese mit dem übrigen Griechenland vergleicht. Mit der seit 1990 liberaleren Minderheitenpolitik in Griechenland scheint sich auch in anderen Bereichen ein - langsamer - Wandel in der Erinnerungskultur anzubahnen, wenn man gewisse Indikatoren so deuten mag wie die Restaurierung des Imaret in Kavala und seine Nutzung als Hotel und Restaurant oder einen ausführlichen Abschnitt über Moscheen und Tekkedes in dem Buch über religiöse Bauwerke in Thrakien (Iera Mitropolis 2005), die Benutzung der Bezeichnungen Türken und türkisch im offiziellen Sprachgebrauch und die Schaffung von Grundlagen für eine schriftliche Erinnerungskultur der Pomaken durch Erstellung eines pomakischen Wörterbuches. Offizielle Erinnerungskultur ist immer auch Politik, die sich mit verändernden gesellschaftlichen Verhältnissen und Machtkonstellationen wandelt.

Man wird, so vermute ich, viele Parallelen zu dieser Form der offiziellen Erinnerungskultur in anderen Balkanstaaten mit junger Geschichte und langer $\mathrm{Zu}-$ gehörigkeit zum Osmanischen Reich feststellen können. Ob und wie die nationale Erinnerungskultur und deren Unterdrückung das Zusammenleben von Christen und Muslimen beeinflusst, muss vorerst dahin gestellt bleiben.

\section{Literaturverzeichnis}

Assmann, Aleida, 1999: Erinnerungsräume. Formen des kulturellen Gedächtnisses. Heidelberg.

Assmann, Jan, 2002: Das kulturelle Gedächtnis. Schrift, Erinnerung und politische Identität in frühen Hochkulturen. München, 4. Aufl.

Avramidou-Kemanetzi, Evlabia A. / Aivaliotis, Basileios D., 2003: Toponymia tis Xanthis [Toponyme Xanthis], Xanthi.

Chatzidimitriou, S., 1992: „I proelevsi ton onomaton tou oikismou tou nomou Xanthis kai i praktiki tis metonomasias tous" [Die Herkunft der Ortsnamen des Nomos Xanthi und die Praxis ihrer Umbenennung], in: Thrakika Chronika 56(1992), 71-88.

Cornelißen, Christoph, 2003: „Was heißt Erinnerungskultur“, in: Geschichte in Wissenschaft und Unterricht 54(2003), $10 \mathrm{ff}$.

Cornelißen, Christoph / Klinkhammer, Lutz / Schwerdtheim, Wolfgang (Hrsg.), 2003: Erinnerungskultur Deutschland, Italien, Japan im Vergleich. Frankfurt.

Der Kleine Pauly. Lexikon der Antike, Stuttgart 1964.

Dimos Xanthis (Hrsg.), o.J.: Xanthi, Thrace-Greece, Broschüre zur Stadt und Umgebung. 
Dimos Komotinis, 1994: Komotini, Analytikos odigos ton odon kai plateion tis polis [Führer zu den Namen der Straßen und Plätze von Komotini], Komotini.

Erll, Astrid, 2005: Kollektives Gedächtnis und Erinnerungskulturen. Eine Einführung. Stuttgart.

Galinos, Alexandros, 1991: „Ta grammatosima tis diasymmachikis (Dytikis) Thrakis“ [Die Briefmarken des umstrittenen Westthrakien], in: Thrakika Chronika, 60-67.

Generalsekretariat der Region Ost-Makedonien (Hrsg.), 1994: Thrakien.

Giannoudi, Dimitriou Ioannou, 1975-76: „Ta grammatosima tis Thrakis“ [Die Briefmarken Thrakiens], in: Thrakika Chronika, Etesia ekdosi No. 32.

Hatschikjan, Magarditsch / Troebst, Stefan, 1999: Südosteuropa. München.

Hering, Gunnar, 1979: „Griechenland vom Lausanner Frieden bis zum Ende der Obristen-Diktatur 1923-1974“, in: Schieder, Theodor (Hrsg.): Handbuch der europäischen Geschichte, 7/2, Stuttgart, 1313-1338 (= Hering, Nostos, 11-47).

Hering, Gunnar, 1989: „Die Osmanenzeit im Selbstverständnis der Völker Südosteuropas“, in: Majer, Hans-Georg (Hrsg.): Die Staaten Südosteuropas und die Osmanen (Südosteuropa-Jahrbuch 19). München, 355-380 (= Hering, Nostos, 327-362).

Hering, Gunnar, 1995: Nostos, Gesammelte Schriften zur südosteuropäischen Geschichte, (Stassinopoulou, Maria A. Hrsg.), Frankfurt a.M., Berlin und a.O.

Iera Mitropolis Xanthis kai Peritheoriou / Moufteia Xanthis, Holy Metropolis of Xanthi and Peritheorion - Mufti of Xanthi (Hrsg.), 2005: Thriskeftika mnimata sto nomo Xanthis / Religious monuments in the prefecture of Xanthi. Xanthi.

Jong, F. de, 1980: „The Muslim Minority in Western Thrace“, in: Ashworth, Georina, (Hrsg.): World Minorities, vol. III, Sunbury (Middlesex, U.K.).

Jong, F. de, 1980a: Names, religions denomination and ethnicity of settlements in W. Thrace. A supplement to the Ortsnamenkonkordanz der Balkanhalbinsel. Leiden.

Jongh, Brian de, 1985: Griechenland. München, 4. Aufl.

Kandler, Hermann, 2007: Christen und Muslime in Thrakien. Berichte aus dem Arbeitsgebiet Entwicklungsforschung 34, Münster.

Karamitsos, A., 2003: Katalogos grammatosimon kai tachydromiki istoria / Stamp Catalog and Postal History. 2 Bde., Thessaloniki.

Kizis, Iannis, 1990: Thraki. Elliniki paradosiaki architektoniki [Thrakien. Griechische traditionelle Architektur]. Melissa, Athen.

Kokkas, N., 1999: Paradosiakoi oikismoi oreinis Xanthis [Traditionelle Siedlungen des Gebirgsraumes von Xanthi]. Xanthi.

Koselleck, Reinhart / Jeismann, Michael (Hrsg.), 1994: Der politische Totenkult. Kriegerdenkmäler in der Moderne. München.

Kreiser, Klaus, 1975: Die Ortsnamen der europäischen Türkei nach amtlichen Verzeichnissen und Kartenwerken, Freiburg.

Kreiser, Klaus, 1978: Die Siedlungsnamen Westthrakiens nach amtlichen Verzeichnissen und Kartenwerken (= Islamkundliche Untersuchungen 51). Freiburg.

Kunz, Josef L., 1925: „Die völkerrechtliche Option“, in: Handbuch des Ausschusses für Minderheitenrecht, Bd. 1. Breslau.

Ladas, Stephen P., 1931: The exchange of minorities Bulgaria, Greece and Turkey. The Macmillan Company. New York. 
Lienau, Cay, 2000: „Die Muslime Griechenlands - zum Problem von Ethnizität, Identität und Nationalität“, in: Lienau, Cay / Steindorff, Ludwig (Hrsg.): Ethnizität, Identität und Nationalität in Südosteuropa. Südosteuropa-Studie 64. München.

Lienau, Cay, 2002: „Staatssymbolik auf griechischen Briefmarken“, in: Choregia, Münstersche Griechenland-Studien 1. Münster, 168-180.

Lipp, Wilfried, 1999: „Denkmalpflege und Geschichte“, in: Borsdorf, Ulrich / Grütter, Theodor (Hrsg.): Orte der Erinnerung. Denkmal, Gedenkstätte, Museum. Frankfurt, New York, 131-168.

Michel Europa-Katalog 1999/2000, 2 Bde. München.

Schultze, Joachim Heinrich, 1937: Neugriechenland. Eine Landeskunde Ostmakedoniens und Westthrakiens mit besonderer Berücksichtigung der Geomorphologie, Kolonistensiedlung und Wirtschaftsgeographie. Gotha (= Petermanns Mitteilungen 233, Erg.-H.).

Stamatelatos, Michail / Stamatelatou, Fotini Bamba, o.J.: Elliniki geografiki enkyklopaideia [Griechische Geographische Enzyklopädie], Ekdoseis TegopoulosManiateas A.E.

Soustal, Peter, 1991: Thrakien (= Tabula Imperii Byzantini 6). Wien.

Sundhaussen, Holm, 2001: „Die Dekonstruktion des Balkanraumes (1870-1913)“, in: Lienau, Cay (Hrsg.): Raumstrukturen und Grenzen in Südosteuropa. München, 19-41 (= Südosteuropa-Jahrbuch 32).

Tzermias, Pavlos, 1994: Die Identitätssuche des neuen Griechentums - eine Studie zur Nationalfrage mit besonderer Berücksichtigung des Makedonienproblems. Freiburg, Schweiz.

Wittek, Paul, 1935: „Von der byzantinischen zur türkischen Toponymie“, in: Byzantion 10, 11-64.

Ypourgeio Politismou, Tameio Archaiologikon Poron kai Apallotrioseon, 2005: Egnatia Odos. Istoria kai Diadromi sto Choro tis Thrakis [Die via Egnatia und ihr Verlauf im Raum Thrakien]. Athen. 
